
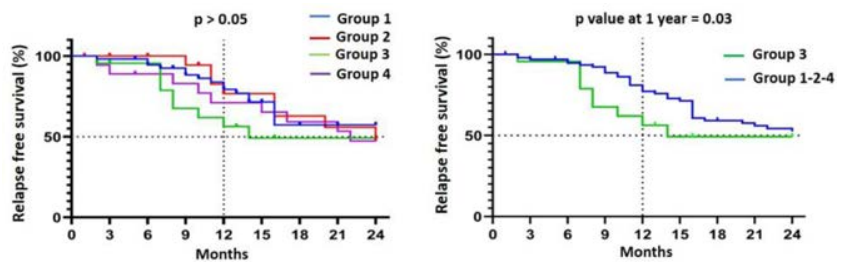

Figure 1. Relapse free survival of the four different IgG4-RD phenotypes.

Conclusion: Clinical phenotypes of IgG4-RD reflect differences in epidemiological features and prognostic outcomes.

References:

[1] Bledsoe JR, Della-Torre E, Rovati L, Deshpande V. IgG4-related disease: review of the histopathologic features, differential diagnosis, and therapeutic approach. APMIS. 2018;126:459-476.

[2] Wallace ZS, Zhang Y, Perugino CA, Naden R, Choi HK, Stone JH; ACR/ EULAR IgG4-RD Classification Criteria Committee. Clinical phenotypes of IgG4-related disease: an analysis of two international cross-sectional cohorts. Ann Rheum Dis. 2019;78:406-412.

Disclosure of Interests: Marco Lanzillotta: None declared, Emanuel Della Torre: None declared, Corrado Campochiaro Speakers bureau: Novartis, Pfizer, Roche, GSK, SOBI, Gaia Mancuso: None declared, Lorenzo Dagna Grant/research support from: The Unit of Immunology, Rheumatology, Allergy and Rare Diseases (UnIRAR) received unresctricted research/educational grants from Abbvie, Bristol-Myers Squibb, Celgene, Janssen, Merk Sharp \& Dohme, Mundipharma Pharmaceuticals, Novartis, Pfizer, Roche, Sanofi-Genzyme, and SOBI., Consultant of: Prof Lorenzo Dagna received consultation honoraria from Abbvie, Amgen, Biogen, Bristol-Myers Squibb, Celltrion, Novartis, Pfizer, Roche, Sanofi-Genzyme, and SOBI.

DOI: 10.1136/annrheumdis-2020-eular.1438

\section{SAT0529 CLINICAL CHARACTERISTICS AND TREATMENT PATTERNS IN A PATIENT GROUP WITH INTERSTITIAL LUNG DISEASE.}

R. Ortega Castro ${ }^{1}$, P. S. Laura ${ }^{1}$, F. U. Pilar ${ }^{1}$, J. Calvo Gutierrez ${ }^{1}$, A. RequejoJimenez $^{2}$, S. Espejo-Pérez ${ }^{3}$, T. Gonzalez-Serrano ${ }^{4}$, M. D. C. Castro Villegas ${ }^{1}$, G. G. Ignacio ${ }^{1}$, A. Escudero Contreras ${ }^{1}$, E. Collantes Estevez ${ }^{1}$, M. A. Aguirre ${ }^{1}$ ${ }^{1}$ Rheumatology Service, Reina Sofia Hospital/IMIBIC/ University of Cordoba, Cordoba, Spain, Córdoba, Spain; ${ }^{2}$ Pneumology Service, Reina Sofia Hospital, Cordoba, Spain, Córdoba, Spain; ${ }^{3}$ Radiology Service, Reina Sofia Hospital, Cordoba, Spain, Córdoba, Spain; ${ }^{4}$ Pathological Anatomy Service, Reina Sofia Hospital, Cordoba, Spain, Córdoba, Spain

Background: Diffuse interstitial lung disease (ILD) is frequently associated with connective tissue diseases (CTD) and is one of the main causes of morbidity and mortality in these patients. Recently, the concept of Interstitial Pneumonia with Autoimmune Features (IPAF) has been defined to characterize ILD associated with systemic manifestations limited to subtle serological and clinical autoimmune abnormalities and not fulfilling the international criteria for the diagnosis of a given CTD.

Objectives: The objective of this study is to describe the clinical, serological and radiological characteristics, as well as the treatment patterns of patients with ILD referred to a Rheumatology Service for suspected CTD

Methods: Observational, cross-sectional study of 43 patients with ILD referred for evaluation to the medical consultation of CTD of the Rheumatology service at the Reina Sofía Hospital. Patients were classified as patients with defined CTD, patient with IPAF and patients with other types of pneumopathy. We conducted a descriptive study of all patients and compared the clinical-analytical-radiological characteristics and treatment patterns of the first two groups.

Results: Of the 43 patients, $67.40 \%$ were women with a mean age at diagnosis of 65.65 (10.42) years and $53.50 \%$ of smoking patients

Of the total of patients, $16(37.2 \%)$ were included in the CTD group, 17 (39.5\%) met criteria for IPAF and 10 (23.3\%) had another type of pneumopathy.

In the CTD group scleroderma was the most frequent disease (6/16), followed by inflammatory myopathy (4/16), Sjögren's syndrome (3/16), rheumatoid arthritis $(2 / 16)$ and polymyalgia rheumatic (1/16). In this group of patients, the most common symptom was Raynaud's phenomenon (RP) (7/16), followed by arthritis (7/16) and mechanic's hands (3/16). Regarding the most frequently antibodies were ANA $(100 \%)$, anti-RO $(41.7 \%)$, anti-citrullinated protein antibodies $(30 \%)$ and rheumatoid factor (RF) (28.6\%).

In patients with IPAF, as in the CTD group, the most observed clinical criterion was RP (5/17), followed by arthritis (1/17) and mechanic's hands (1/17). Among the serological criteria the most common antibodies were ANA (100\%), followed by anti-RO (33.3\%), anti-RNA synthetase (28.6\%) and RF (22.2\%).
Regarding the radiological pattern, in both groups the most frequent was nonspecific interstitial pneumonia, followed by the indeterminate pattern and usual interstitial pneumonia (UIP) in third place. There were no significant differences by gender and age, between the group of CTD and IPAF, observing in both groups a predominance of women with a similar mean age, being the upper smoking habit in the IPAF group $(70.6 \%$ vs $31.5 \%, p=0.02)$. Regarding the treatment used, the use of immunosuppressants (IS) was more frequent in CTD group $(56.3 \%$ vs $11.8 \%, p=0.007$ ).

Conclusion: The clinical-serological and radiological characteristics were similar among patients with IPAF and CTD, which supports the notion of a similar pathophysiology in both groups. In our cohort patients with CTD received IS more frequently than IPAF group, however, future work would be necessary to assess whether the response to treatment is similar in these populations and if IS can benefit patients with IPAF to long term. In addition, it could be useful to include the UIP pattern within the IPAF classification criteria, not currently included, since it is the third most frequent radiological pattern.

\section{References:}

[1] Respirology, 21 (2016), pp. 245-258

[2] Eur Respir J, 46 (2015), pp. 976-987

Disclosure of Interests: Rafaela Ortega Castro: None declared, Pérez Sánchez Laura: None declared, Font Ugalde Pilar: None declared, Jerusalem Calvo Gutierrez: None declared, Antonio Requejo-Jimenez: None declared, Simona Espejo-Pérez: None declared, Teresa Gonzalez-Serrano: None declared, María del Carmen Castro Villegas: None declared, Gómez García Ignacio: None declared, Alejandro Escudero Contreras: None declared, Eduardo Collantes Estevez Grant/research support from: ROCHE and Pfizer, Speakers bureau: ROCHE, Lilly, Bristol and Celgene, Maria A Aguirre: None declared DOI: 10.1136/annrheumdis-2020-eular.5812

\section{SAT0530 \\ DESCRIPTION AND OUTCOMES OF A SERIES OF 21 PATIENTS WITH INTERSTITIAL PNEUMONIA WITH AUTOIMMUNE FEATURES IN A MULTIDISCIPLINARY UNIT.}

M. D. C. López González¹, E. Nofuentes', S. Ruíz Alcaraz', I. Gayá GarcíaManso $^{1}$, R. García Sevila ${ }^{1}$, P. Vela-Casasempere ${ }^{1}$, M. Andrés ${ }^{1} .{ }^{1}$ Hospital General Universitario De Alicante, Alicante, Spain

Background: A significant proportion of patients with idiopathic interstitial pneumonia shows autoimmune features, but do not meet the criteria to be classified as a systemic autoimmune disease. In 2015, a joint working group of the European Respiratory Society (ERS) and the American Thoracic Society (ATS) proposed classification criteria for the category called interstitial pneumonia with autoimmune features (IPAF).

Objectives: To evaluate the clinical, serological and morphological characteristics of a series of 21 patients with IPAF as well as the treatment that was applied and the evolution they presented.

Methods: A retrospective cohort study was conducted. The patients who met ERS/ATC IPAF classification criteria in the period from 2012 to 2019 were collected from our interstitial lung disease (ILD) database, including 546 patients. All cases were systematically assessed in a multidisciplinary committee. Clinical, serological, morphological, as well as treatment and outcome variables were collected. A Descriptive analysis is shown.

Results: 21 patients were included in the study, 12 of them $(57.1 \%)$ women. The mean age at diagnosis was 61.6 years (SD 14.0), and the median follow-up time was 2.9 years (IQR 4.9). All showed ILD by HRCT, 10 (47.6\%) patients had autoimmune features at the moment of the diagnosis, and 20 (95.2\%) were positive for some auto-antibody. $12(57.1 \%)$ fulfilled two of the three domains, and $9(42.9 \%)$ fulfilled the three IPAF domains. Characteristics are described in Table 1. Lung biopsy was performed in 9 cases (42.9\%). The mean FVC at diagnosis was $70.4 \%$ (DS 21.0) and DLCO $46.7 \%$ (DS 21.5). Regarding the treatment during the disease, mofetil mycophenolate (MMF) was used in $8(38.1 \%)$ patients, cyclophosphamide (CYC) in $3(14.3 \%)$, rituximab (RTX) in $2(9.5 \%)$ and azathioprine in $2(9.5 \%)$. In 1 case $(4.8 \%)$, CYC and RTX were used in combination. Oral glucocorticoids (GCC) were prescribed in 14 patients $(66.7 \%)$, 4 of them alone, and 6 cases (28.6\%) required high-dose methylprednisolone in 250-1000 mg regimens. Antifibrotic drugs (nintedanib or pirfenidone) were used in 5 cases $(23.8 \%)$ in combination with immunosuppressants and/or GCC. Five patients $(23.8 \%)$ required home oxygen supplementation. Outcomes to date: $7(33.3 \%)$ patients had readmissions due to ILD acute exacerbation; $2(9.5 \%)$ required lung transplantations, and $3(14.3 \%)$ died during follow-up, $1(4.8 \%)$ due to respiratory causes. 